\title{
Per un approccio comunicazionale al logos sokratikos: Le Dialogue socratique di Livio Rossetti
}

\author{
Cristiana CASERTA \\ Università degli Studi di Palermo \\ cristiana.caserta@tin.it
}

Accade che polverose quaestiones storico-filologiche o discussioni accademiche su singoli, minuscoli aspetti di un testo o di un corpus di opere subiscano bruscamente uno scarto che le pone su nuove direttrici di ricerca e schiude campi intatti, laddove parevano esservi solo angusti orizzonti compilativi.

È quanto è avvenuto, dagli anni '90 del secolo ormai scorso, agli studi socratici, di cui si segnala una vera e propria "renaissance", come indica il numero ormai consistente di momenti di confronto e di dibattito: dal forum periodico Socratica, giunto nel 2012 alla sua terza edizione, ai molti colloqui e simposi dedicati a singoli autori. ${ }^{1}$

I frutti di questa nuova temperie culturale includono una serie di studi sul dialogo socratico inteso anche come genere letterario, fra i quali spiccano i contributi di Donald Morrison, Louis-André Dorion, Michel Narcy $^{2}$ e in primissimo luogo di Livio Rossetti, la cui ultima pubblicazione Le Dialogue socratique (cf. p. 239), raccoglie otto saggi di argomento socratico scritti fra il 1998 e il $2010 .^{3}$

Il volume è una preziosa sintesi dei risultati raggiunti da Rossetti nel campo degli studi socratici e, al contempo, un fondamento del loro ulteriore sviluppo. Da un lato, infatti, lo studioso definisce una serie di problemi e di metodi utili a delimitare il campo: il dialogo socratico in quanto genere letterario, la sua nascita come avvenimento culturale di straordinaria portata, l'esistenza di unità dialogiche a se stanti all'interno dei dialoghi platonici e dei Memorabilia di Senofonte e la correlata necessità di studiarle come tali, l'individuazione di una imitatio Socratis relativa alla pratica dell'elenchos, la forza comunicativa del ridicolo, il problema della retorica socratica, il metodo che Rossetti chiama di 'distillazione' degli elementi di contesto per isolare e analizzare i punti di

${ }^{1}$ Un quadro delle recenti tendenze degli studi socratici in Stavru 2005; Rossetti 2008; Stavru-Rossetti 2010a.

${ }^{2}$ Vd. Morrison 1988; Dorion 2000; Narcy 2007; Rossetti 2011.

${ }^{3}$ Si tratta di una scelta di alcuni fra i più significativi, dei moltissimi lavori di Rossetti dedicati a Socrate (cf. p. 239, n. 1). 
dottrina, per citarne alcuni; dall'altro, i risultati che egli presenta sono indiscutibilmente nuovi ma anche sufficientemente aperti da lasciare un margine di ulteriore dibattito.

Il presente contributo si articola pertanto in due momenti: la ricognizione del campo degli studi di Rossetti, organizzata intorno ad alcuni temi generali (il dialogo socratico, il dialogo aporetico, Socrate in azione, la retorica socratica, l'inautenticità del dialogo platonico) e la valutazione delle aperture verso 'nuove' questioni.

Poiché, come spesso accade a quegli studiosi che producono lo scarto di cui dicevamo, è un problema apparentemente marginale a catturare l'attenzione di Rossetti e a tale problema lo studioso non ha cessato di dedicare le sue energie in un impegno più che trentennale (cf. supra, p. 241), conviene, per rendere giustizia allo spessore del libro, risalire a quella radice lontana. Il problema si presenta, in uno scritto del 1974, ${ }^{4}$ sotto questa forma: aspetti particolari e interessanti (e dissonanti dalla vulgata filosofica corrente) della personalità di Socrate figurano ascritti al filosofo in fonti comunemente reputate di scarso valore, in aneddoti, apoftegmi, gnōmai, argomentazioni apologetiche che si trovano disseminate in tutta la letteratura di età ellenistica e posteriore. Ciò induce a ritenere i Socratica platonici e senofontei insufficienti per una conoscenza adeguata della personalità di Socrate e a supporre che informazioni preziose debbano trovarsi in altre fonti, magari non pervenute fino a noi, in particolare nei molti logoi sokratikoi apparsi nel IV secolo a. C.

E qui, nello studio del '74 iniziavano le difficoltà, perché mancava una silloge dei testi di tutti i Socratici minori (lacuna che sarà colmata solo nel 1983-85 dalle Socraticorum Reliquiae del Giannantoni e poi dalla successiva edizione ${ }^{5}$ ), si cominciavano appena ad editare i frammenti di Aristippo e dei Cirenaici, di Antistene, e dei Megarici, mentre si dovevano ancora attendere parecchi anni per un ripensamento dei socratica senofontei. ${ }^{6}$

Si profilava comunque in tal modo sia «la possibilità di "promuovere" un certo numero di testimonianze seriori dall'attuale condizione di testimonianze svalutate e scarsamente attendibili al ruolo di testimonianze

${ }^{4}$ Rossetti 1974, pp. 424-438.

${ }^{5}$ Vd. Giannantoni 1990.

${ }^{6}$ Una breve storia degli studi filologici e delle edizioni critiche dei Socratici Minori è contenuta in Rossetti 2008a, pp. 11-12 e 16-17 (su Senofonte). 
non meno degne di attenzione e non meno autorevoli di quelle più celebrate di un Platone o di un Senofonte, in quanto approssimativamente coeve, e risalenti a qualcuno degli altri Socratici»; sia quella «di recuperare in tal modo alcuni validi interlocutori ai soliti Platone, Senofonte, Aristofane, Aristotele ecc. nella ricostruzione della figura storica di Socrate». ${ }^{7}$

«Interlocutori» e «coeve» sono le parole chiave per intendere la rotazione di centoottanta gradi nello studio dei Socratici operata da Rossetti: oltre che come fondatori di scuole, secondo lo schema di Diogene Laerzio, i Socratici sono finalmente visti come contemporanei di Platone e, come lui, allievi di un comune maestro. ${ }^{8}$

Il frutto di questa rivoluzione è ora contenuto nei due saggi posti significativamente in apertura e in conclusione del libro: "Le dialogue socratique in statu nascendi" (Cap. 1) e "Le socratiques "premiers philosophes» et Socrates «premier philosophe»" (Cap. 2). Essi costituiscono una trattazione unitaria del fenomeno collettivo cui diede vita il gruppo degli allievi di Socrate, includendo in questo novero anche autori mal conosciuti come Euclide, Critone, Glaucone, Alessameno di Teo. A questo gruppo, nel suo insieme, va fatta risalire una serie cospicua di invenzioni e innovazioni, fra cui la nascita del dialogo socratico come genere letterario nuovo; la creazione di una mentalità nuova e di nuovi modi di insegnamento, la produzione di «une idée fort caracterisée (et à plusiers égards inédite) de ce que signifie philosophein». ${ }^{9}$

\section{Il dialogo socratico}

L'aver visto il dialogo socratico come genere letterario, con una sua consistenza, un suo ciclo vitale e un suo 'dna', non è intuizione di poco

${ }^{7}$ Rossetti 1974, p. 425.

${ }^{8}$ Un ripensamento in tal senso era stato stimolato dalla pubblicazione, nel 1990 , dell'imponente raccolta di fonti e dati costituita da Socratis et Socraticorum Reliquiae a cura di G. Giannantoni. Dai dubbi del Giannantoni sulle scuole socratiche nacque infatti una riflessione sull'opportunità di adottare un criterio sincronico anziché diacronico e si cominciò a focalizzare l'attenzione sulla circostanza inoppugnabile che la prima generazione di allievi di Socrate dovette condividere con Platone molto di più di quanto non si fosse pensato fino ad allora. In particolare, quella generazione di intellettuali condivideva la prassi di riprodurre le discussioni avute col maestro in forma di dialoghi. I primi segni di questa nuova impostazione sono in Kahn 2008 (1996).

${ }^{9}$ Rossetti 2011, p. 24. 
conto, né di scarse conseguenze. Il 'dialogo socratico', nell'intepretazione comunicazionale di Rossetti, non è infatti esattamente sovrapponibile, per temi e struttura, ai primi dialoghi di Platone, quelli comunemente chiamati 'socratici'. Il formato più autentico del genere letterario identificato da Rossetti è invece l'unità dialogica, che poteva costituire un libro o rotolo a se stante, ovvero l'articolazione, con altre unità, di un'opera più complessa. Queste unità, ha calcolato lo studioso, dovevano essere circa trecento, prodotte e pubblicate fra il 394 e il 370 a.C. Una produzione enorme, che non poté certo passare inosservata nell'Atene del tempo e che è anche il sintomo di un consistente bisogno di scrittura come modo per non disperdere l'eredità culturale del maestro, anche da parte di allievi poco dotati come scrittori. A costoro potrebbe infatti risalire un certo numero di logoi anonimi sopravvissuti in forma frammentaria (aneddoti, khreiai, apoftegmi).

Ancora più importante è, a me pare, l'aver indicato l'origine del genere letterario in una pratica "endosocratica", evidenziandone la discontinuità rispetto a modelli di dialogo già esistenti all'interno di altri generi letterari: storiografia, commedia, mimo. La sua proposta è di ravvisarne l'origine non in quei precedenti ma nel processo di standardizzazione del dialogare socratico, con le sue modalità, i suoi temi, i suoi effetti emotivi sull'interlocutore: un modo peculiare di comunicare messo a punto, abitualmente usato e progressivamente affinato da Socrate stesso e poi appreso e messo in pratica anche dagli allievi più vicini al maestro. «On a donc - sostiene lo studioso - des raisons de voir dans ces usages à l'intérieur de la communauté formée par Socrate set ses élèves (sourtout, ou au moins, vers la fin de la vie du philosophe) un logos Sōkratikos in statu nascendi». ${ }^{10}$

Con ciò si dice molto di più di quanto appaia, perché si affida alla stesura per iscritto del dialogo la funzione di una strategia transitoria all'interno di un evento comunicativo molto più ampio e complesso. Il testo, che non va confuso con quella che è la sua fissazione per iscritto, dovette essersi formato nel tempo e nell'interazione reale e solo in seguito - più o meno occasionalmente - essere stato costituito come testo scritto, senza tuttavia che ciò fosse lo scopo originario o unico dell'evento comunicativo. La trascrizione è dunque più il resoconto di un processo comunicativo, che il risultato di quel processo: una prassi assai

${ }^{10}$ Rossetti 2011, p. 44. 
lontana da quella che oggi intendiamo per 'pubblicazione' di un testo. «Le logos Sokratikos - afferma Rossetti- a les traits, non du récit, mais de la recréation efficace d'une telle dynamique, de sa mise en oeuvre et des effets à atteindre». ${ }^{11}$ Una dinamica al cui interno Rossetti ricostruisce vari momenti di costruzione del testo, a cui partecipavano tanto gli allievi quanto il maestro e che comprendevano l'improvvisazione orale di un tipico dialogo socratico (per uditori estranei), la redazione scritta di una conversazione già svolta, sulla base di appunti o di informazioni raccolte e di controlli effettuati, la preparazione in vista del racconto, il racconto orale vero e proprio - una sorta di conferenza - davanti ad uditori. A questo complesso di abitudini e pratiche comunicative, la morte di Socrate dovette imprimere una rapida svolta in ordine alla volontà sia di preservare l'immagine del maestro, sia la lettura che la comunità degli allievi dava degli avvenimenti precedenti la sua morte.

Veniamo così, dall'esplorazione delle forme di scrittura e di memoria, al riconoscimento di un nucleo identitario che tali strumenti erano chiamati a salvare: un'autorappresentazione collettiva tale da rendere riconoscibile la comunità dei suoi allievi, oltre la morte del maestro. È merito di Rossetti aver identificato gli elementi di una simile identità basata sulla partecipazione ad un sapere e una memoria comuni e aver descritto, riesaminando le fonti sul termine 'filosofia', il processo che tendeva alla autodefinizione di Socrate come 'filosofo' e dei Socratici come 'primi filosofi' (cf. supra, p. 270).

La fase iniziale di questo processo sembra essere stata l'idea di una contrapposizione fra politici e filosofi, che Rossetti ritiene legata agli sviluppi della speculazione platonica. Socrate e i suoi interlocutori condividono «une idée assez précise de la philosophie», ${ }^{12}$ come qualcosa che accade, che può far notizia, che ha una consistenza oggettiva, se il maestro può essere rappresentato nel Carmide, nell'atto di chiedere: «che novità ci sono riguardo alla filosofia». ${ }^{13}$ Analoghi riferimenti si trovano in Senofonte, non solo come fenomeno proiettivo, cioè retrodatazione agli ultimi decenni del v secolo di un uso linguistico in realtà affermatosi solo più tardi (dopo il 399 a.C.); ma anche in relazione a precisi fatti storici nel periodo dei Trenta Tiranni, in cui i filosofi costi-

\footnotetext{
${ }^{11}$ Rossetti 2011, p. 44.

12 Rossetti 2011, p. 268.

${ }^{13}$ Pl. Charm. $153 \mathrm{~d}$.
} 
tuiscono un gruppo con una precisa identità collettiva. Sia Senofonte che Platone infatti indicano la circostanza della presa di potere da parte dei Trenta come momento in cui era abituale per la gente comune scagliarsi contro i filosofi con ingiurie che, in mancanza di precise accuse, venivano lanciate anche contro Socrate. ${ }^{14}$

Una seconda fase, che supera la visione puramente pregiudiziale e stereotipata dei filosofi, è quella di stabilizzazione dell'uso del termine 'filosofo' e 'filosofia' ad opera dei Socratici che si può collocare nei primi decenni del IV secolo. I Socratici, secondo l'interpretazione comunicazionale, facevano inequivocabilmente riferimento, nei dialoghi da essi scritti in enorme quantità, ad un esercizio del filosofare che avevano conosciuto frequentando Socrate.

A questa fase risalgono tre cospicue novità:

l'utilisation extensive du terme de philosophie, la représentation de personnes en train d'élaborer des idées, et la puissance de la 'loupe', entièrement inédite, que Platon sut mettre au point pour représenter le premier philosophe et ses interlocuteurs aux prises avec les embûches de l'acte de philosopher. ${ }^{15}$

Nasce cioè un modo specifico di rappresentarsi come 'filosofi', e 'filosofia' diventa una etichetta in cui convergono tanto l'attitudine ad un movimento verso la sophia piuttosto che al possesso di essa, quanto la rappresentazione - nei dialoghi socratici- del filosofo 'in azione', nonché la riproduzione della riflessione filosofica mentre sta avendo luogo.

\section{Un contesto per il dialogo aporetico}

Quali conseguenze derivano da questa serie di scoperte?

In primo luogo, esse chiamano in causa i "Socratici Maggiori", Platone e Senofonte, la cui interpretazione complessiva deve essere ricalibrata.

Per quanto concerne Senofonte, occorre sottolineare che da almeno due decenni è in atto una riscoperta dei Socratica senofontei che ha portato ad un sostanziale scioglimento delle riserve gravanti per tradi-

${ }^{14}$ X. Mem. I.6.2; I.2.31; Pl. Apol. $23 \mathrm{~d}$.

${ }^{15}$ Rossetti 2011, p. 276. 
zione sulle capacità di scrittore e di filosofo di quello che è sempre stato considerato al più un fratello minore del grande genio, Platone. ${ }^{16}$ Per contro, nell'interpretazione comunicazionale, è importante trovare «ce qui fait l'identité de la façon xénophontienne d'aborder des sujets philosophiques (et non pas seulement historiques) et notamment de traiter de Socrate», giungendo per questa via ad apprezzare il valore di alcuni passaggi senofontei come «témoignage sur la façon typiquement socratique de pratiquer l'elenchos et sur ce qui a pu être coutumier à l'intérieur de son cercle d'amis-élèves». ${ }^{17} \mathrm{Si}$ vedrà più avanti quale sia questo valore.

Ma il riconoscimento dell'esistenza del logos sokratikos produce un forte impatto soprattutto sugli studi platonici.

Anzitutto, occorre pensare che Platone dovette attingere gradualmente una posizione di prestigio nella cerchia degli allievi di Socrate, posizione che certamente non aveva agli inizi degli anni '90 del IV secolo a.C. , quando verosimilmente cominciò come ad essere conosciuto come scrittore di dialoghi: «understanding Plato's ideas means therefore dwelling on the context in which they developed and eventually emerged as the most prominent». ${ }^{18} \mathrm{E}$ bisogna anche chiedersi se una dinamica di tal genere non comporti anche per Platone, in quanto scrittore di dialoghi socratici, un "taglio" peculiare, una scelta precisa di alcuni tratti della personalità del maestro da esemplificare nei propri scritti a scapito di altri da lasciare in ombra. Uno sguardo sinottico all'insieme delle immagini del maestro costruite dai Socratici, incluso Platone, può chiarire la differenza fra il Socrate 'agente' per conto di Platone, argomentatore di teorie e produttore di dottrine, e il Socrate 'in azione' per proprio conto. Sostanzialmente identico a se stesso, quest'ultimo, in un gran numero di unità dialogiche, prodotte da allievi con differenti attitudini speculative e variegate posizioni dottrinali

a Socrates - come afferma altrove Rossetti- who happens to be imitated (as in Xen. Mem. III 8 and elsewhere) as well as put aside when he is treated, rather, as the bearer of definite points of doctrine. It will be argued that, once identified such a 'Standard Socrates', the otherwise thick "literary barrier" represented by the dialogues becomes remarkably transparent, suit-

\footnotetext{
${ }^{16}$ Rossetti 2007a, p. 36.

${ }^{17}$ Rossetti 2011, pp. 101-102.

${ }^{18}$ Stavru-Rossetti 2010, p. 16.
} 
able to be crossed ahd therefore to bring us very close to the real, historical Socrates. ${ }^{19}$

Vi è poi la necessità di cercare un contesto per quei dialoghi che una lunga tradizione definisce 'aporetici'. Il saggio centrale del libro (Cap. 2) è dedicato a due problemi: contestualizzazione del dialogo aporetico e ricerca dello specifico dell'Eutifrone, attraverso l'analisi testuale. ${ }^{20}$

Cominciamo da quest'ultimo punto, che in realtà è affrontato per ultimo nel libro, per dare meglio l'idea di come ciò che individua ciascun dialogo sia in definitiva la punta di un vasto iceberg, costituito da ciò che esso ha in comune col resto dell'opera platonica, con la tipologia del dialogo aporetico e con la letteratura socratica nel suo insieme. La lezione primaria dell'Eutifrone (e di altri dialoghi aporetici) si configura, afferma Rossetti, come un «paquet de messages» cui Platone «s'efforce d'accorder droit de cité». ${ }^{21}$ Essi si articolano intorno al tentativo di «faire le point sur la nature des notions que nous utilisons quotidiennement, leur logique interne et le critère qui, à un niveau intuitif, nous permet d'accéder à l'idée qu'il n'est pertinent de faire appel à la notion singulière que pour un répertoire donné de cas». ${ }^{22} \mathrm{Si}$ tratta allora di portare alla luce il criterio di applicazione latente in una nozione che intuitivamente reputiamo pertinente o non pertinente a singoli casi: la pietà, nel caso dell'Eutifrone ; $^{23}$ tentativo che mostra lo iato fra le competenze tecniche, implicanti l'uso spontaneo di una certa nozione, e la capacità di precisare in cosa quella nozione consista, che cosa esattamente indichi, etc.; e che solleva un velo sulle strutture portanti degli usi linguistici e della stessa realtà. Da cui deriva la necessità di raffinare i concetti e prende-

${ }^{19}$ Rossetti 2004, pp. 81-94.

${ }^{20}$ I dialoghi 'aporetici' di Platone sono quelli in cui non sono esposte dottrine e in cui la conversazione non approda ad alcuna certezza sulle questioni indicate come importante dagli interlocutori e da Socrate stesso. Vd. Rossetti 2011, p. 140-169 e 180-194.

${ }^{21}$ Rossetti 2011, p. 182. A margine di queste osservazioni, Rossetti menziona anche, come peculiarità dell'Eutifrone, «d'un côté une part de scepticisme en matière de religion olympique, de l'autre le 'refoulement' de la dimension protreptique».

${ }^{22}$ Rossetti 2011, p. 183.

${ }^{23}$ Una collocazione del dialogo verso la fine della stagione dei dialoghi aporetici porterebbe a ritenere, secondo Rossetti, che Platone non sentisse più l'urgenza di rivendicare i meriti specifici del maestro o la sua religiosità di fondo, che emerge in modo obliquo e discutibile nel dialogo, e si sentisse invece libero di avanzare istanze conoscitive proprie. 
re coscienza di incongruenze e fattori in grado di smentire l'enunciato sotto esame. Ciò accade attraverso l'elenchos dell'interlocutore e anche con l'aiuto di una serie di concetti-chiave, che vengono messi a punto e contemporaneamente insegnati nel corso del dialogo.

L'esistenza di un contesto culturale preciso, in termini di pubblico e di lettori, di un clima di competizione fra socratici per accreditarsi come l'erede più autentico di Socrate, della necessità di una rilettura in termini elogiativi della complessa personalità del maestro, incide naturalmente nella valutazione delle opere della prima maturità di Platone, più che nelle altre. Ciò significa che un dialogo come l'Eutifrone deve essere considerato non solo come precedente alla fondazione dell'Accademia e alla elaborazione del complesso edificio dottrinale della Repubblica e degli altri dialoghi della maturità e della vecchiaia (idee, anima, anamnesi, immortalità); ma soprattutto come esemplare di un genere letterario il cui impatto provocava, anche per la sua novità, una fortissima emozione nel pubblico. Esso dovette costituire una sorta di terza via fra tragedia e commedia, in cui si parlava di attualità con naturalezza e si rappresentavano in giro persone reali, rievocandone l'ambiente e i tratti anche in modo caricaturale, ma si chiedeva allo spettatore/ascoltatore di prendere sul serio le idee che vi si esponevano.

Questi aspetti del genere letterario ed altri che riguardano la riconoscibilità di Socrate - il gusto per le analogie banalizzanti e per i contro esempi, la strategia complessiva con cui porre a proprio agio l'interlocutore per poi chiuderlo nell'aporia, la dichiarazione di non sapere- costituiscono il nucleo forte di quello che Rossetti felicemente chiama il 'Socrate in azione' che i dialoghi socratici rappresentano.

Altri aspetti del dialogo aporetico, secondo l'interpretazione comunicazionale, possono essere invece ascritti al solo Platone: fra questi c'è - e l'Eutifrone lo documenta - la ricerca definitoria, cui non fanno riferimento, afferma Rossetti, né Senofonte né gli altri Socratici. Platone

a conçu et introduit dans ses textes [la recherche définictionelle] en se fondant sur l'habitude qu'avait Socrate de recourir à des questions épisodiques du type 'Qu'est que c'est que X?' lorsqu'il se proposait de susciter chez son interlocuteur un besoin impérieux de changer de vie et de se donner un standard éthique différent de (et nettement supérieur à) celui qui lui était coutumier. ${ }^{24}$

${ }^{24}$ Rossetti 2011, pp. 139-140. 
Sebbene singole tessere della ricerca definitoria possano essere fatte risalire a Socrate, la sua ideazione e impostazione, e le nozioni cardine risalgono, secondo Rossetti, a Platone.

L'interpretazione comunicazionale si fonda sulla ridefinizione di alcune 'regole del gioco' del dialogo aporetico. In primo luogo, una struttura e dei principi di organizzazione che è bene conoscere anche per poter riconquistare una distanza critica. Oltre a mettere in campo Socrate in azione, questo genere di dialogo mette in campo anche un forte squilibrio fra il filosofo e il suo interlocutore, che puntualmente porta alla capitolazione di quest'ultimo. La strategia con cui vi si giunge -dopo un altalenare di emozioni: dapprima un certo senso di superiorità e la speranza di far bella figura con Socrate, poi la percezione netta della difficoltà del compito, l'illusione di poter ancora cavarsela e infine la sconfitta definitiva - induce, nel fruitore del dialogo, il desiderio di trovare lui la chiave dell'enigma; ma anche la consapevolezza che ciò può accadere solo con l'aiuto di Socrate e del suo interprete Platone.

La gestione delle emozioni dell'interlocutore ha dunque un ruolo notevole nell'economia del dialogo, sia nell'indirizzare il dialogo stesso verso uno e un solo esito: la capitolazione dell'interlocutore; sia nell' anticipare sistematicamente le reazioni del lettore.

La peculiarità del congegno ideato da Platone per ottenere questo effetto, avere riconosciuto e descritto il quale è anche uno dei risultati più originali prodotti dall'interpretazione comunicazionale, è il suo essere una forma mista di due 'universali' narrativi, quello patetico e quello comico. Il primo si struttura in due momenti: l'identificazione psicologica fra personaggio principale e lettore e una serie di peripezie da affrontare prima del definitivo scioglimento della tensione. L'universale comico delinea una situazione analoga, ma le peripezie che l'eroe comico deve affrontare sono in genere causate dalla sua stessa insipienza o goffaggine cosicché l'identificazione del lettore risulta stemperata e costui può ridere della vicenda come se non lo riguardasse. Dall'intrecciarsi di queste due strategie narrative, scaturisce quella che si può chiamare l'ambiguità del dialogo aporetico e che, più propriamente, consiste nella presenza di un dispositivo capace di mobilitare una ingentissima quantità di risorse intellettuali da parte del lettore; le quali non sarebbero state rese disponibili se egli avesse potuto sapere sin dall'inizio quale tipo di narrazione, se patetica o comica, lo attendeva. 
Egli, invece, il lettore, non è affatto preparato (e non lo è neppure dopo diverse letture) a ciò che troverà. Anche perché lo attende una vera e propria esperienza intellettuale, 'attiva' e dunque tale da superare i meccanismi dell'empatia, rompere per così dire l'illusione narrativa, per inondare la sua stessa vita; il lettore si trova impegnato a proseguire per proprio conto la ricerca di cui ha intravisto il punto di arrivo senza poterlo, per la durata del dialogo, attingere.

L'inaccessibilità del punto di arrivo è garantita d'altra parte da una serie di vincoli ben studiati da Platone, una serie di requisiti per la definizione che si cerca, tali da condannare chi vi si cimenti ad una serie infinita di micro e macro fallimenti (a seconda della sua attitudine filosofica):

la disproportion entre la définition optimale qu'on recherche et les définitions singulières qui sont proposée au fur et à mesure (le long d'un axe allant de l'approximatif à l'adéquat) est telle qu'elle passionne à coup sûr et exacerbe la capacité d'implication de l'interlocuteur (et, ce qui est plus important, du lecteur), autrement dit elle se fait le véhicule de valeurs communicationnelles de qualité. ${ }^{25}$

Volgendosi dal versante emotivo ed esperienziale a quello del giudizio di merito, e dunque all'interpretazione del dialogo, Rossetti ha modo di rivedere alcuni teorie sull'aporia platonica. Nei dialoghi aporetici - questa la sua idea - fu prioritario per Platone l'aver fissato una metodologia, senza la pretesa di arrivare a risultati definitivi: ciò spiega il gusto con cui, avviata la ricerca definitoria, egli procede a impostare ricerche complesse senza chiudere il cerchio o lasciando che finisca per affiorare soltanto un abbozzo di definizione.

Per il lettore, anche il più attento, è molto difficile formulare un giudizio di merito su un dialogo con l'Eutifrone: egli ne è ostacolato dal meccanismo condizionante per cui, in virtù di una serie di emozioni suscitate in lui, è messo in condizione di abbassare troppo facilmente la guardia, quando Socrate argomenta o eccepisce. «Cela entraîne - conclude l'autore - un épointement de ses capacités analytiques, qui n'est que momentané, certes, mais qui menace aussi l'ensemble des lecteurs». ${ }^{26}$

\footnotetext{
${ }^{25}$ Rossetti 2011, pp. 160-161.

${ }^{26}$ Rossetti 2011, p. 174.
} 
Per il commentatore è facile persuadersi che il discorso 'fila' e partire dal presupposto che Platone non può «être pris en défault», ricorrendo ad argomenti talora inefficaci o controproducenti ma tutto sommato ben mimetizzati nella generale linearità e plausibilità dell'insieme. Un effetto contrario fa apparire le posizioni di Eutifrone più fragili e più inconsistenti di quanto non siano in realtà e al contempo distoglie troppo facilmente il lettore dal proseguire certe linee teoriche abbandonate alquanto frettolosamente.

Rossetti coglie qui con pienezza il frutto del paziente lavoro di indagine sulle emozioni e sulle strategie platoniche - che credo sia valsa la pena seguire in dettaglio - perché è solo decondizionando se stessi dalla suggestione che si può cominciare il lavoro analitico e riguadagnare la distanza critica necessaria. Il dialogo aporetico non chiede soltanto di vivere un'emozione ma chiede, sottolinea lo studioso, il libero assenso dell'intelligenza del lettore: libertà che si raggiunge a patto di capire perché si viene indotti a reagire in certi modi e non in altri.

Il problema di una 'retorica', o come suggerisce Rossetti di una 'inautenticità' di Platone, è posto con forza dall'autore. Negli ultimi decenni, molti commentatori hanno notato le reticenze, le discontinuità dell'argomentazione, il modo obliquo in cui certi enunciati, nei dialoghi platonici, suggeriscono il senso (o un senso) complessivo dell'intero evento comunicativo. Questo "côté 'para-énonciatif'» del dialogo platonico è stato esplorato, ma molto resta ancora da fare. Soprattutto se si pensa che certe 'zone oscure' della comunicazione platonica non sempre e necessariamente devono essere interpretate come evocative di dottrine non scritte. Alcuni messaggi - argomenta Rossetti- sono semplicemente «inépuisables ou insondables», ovvero ben protetti. A funzionare da protezione è il 'mélange' di elementi dottrinali ed elementi emozionali, di appelli all'esercizio di critica e di inviti sottili all'identificazione empatica. Ma non soltanto: sollecitazioni inavvertite alla sospensione del controllo di merito su alcuni enunciati e non su altri si nascondono in parti apparentemente innocue del dialogo, nelle formule di transizione da un argomento all'altro, negli incisi, nelle locuzioni avverbiali con cui l'interlocutore dà l'assenso ai ragionamenti del filosofo. E così anche certe locuzioni apparentemente neutre fanno invece da robusto argine al contenimento dell'argomentazione entro ben precisi limiti. Per non dire delle volte in cui a sostenere 'plasticamente' il ragionamento è la defaillance dell'interlocutore o la sua eccessiva docilità, che funziona 
talora da deus ex machina facilitando il trionfo di un argomento non irresistibile.

F. Roustang, nella prefazione del volume, racconta di aver pensato, leggendo questo saggio, che «quelque chose de peu ordinaire avait fait irruption dans le petit monde des études platonicienne» e afferma che con esso Rossetti ha inventato un nuova chiave di lettura. Una chiave, si può aggiungere, universale. Perché, negando che si dia come oggetto di studio l'argomento 'nella sua nudità', e che si possa accedervi 'ritagliandolo' dal contesto, come una silhouette da uno sfondo, l'interpretazione comunicazionale di Rossetti pone un'obiezione di fondo sia all'approccio 'esoterico' della scuola di Tubinga, sia a quello analitico degli studiosi americani e mette le basi di una ricerca sottratta a questa o quella prospettiva ermeneutica.

Quello sfondo, che è invece un contesto pervasivo e potente, è infatti per Rossetti il connettivo che consente all'interprete di integrare, esplicitare, far parlare un testo reticente, allusivo, frammentario. Non si può attingere la dottrina, anche non esoterica, se non si identifica, si analizza e infine si neutralizza il filtro protettivo che il contesto dialogico frappone: ecco i termini di una 'questione platonica' del tutto originale. Ed ecco anche la prospettiva di lavoro: mettere a punto gli strumenti e la procedura per una 'distillazione' di un simile resistente mélange.

\section{Un altro Socrate (a partire da Senofonte)}

Il Socrate in azione, prediletto dall'interpretazione comunicazionale, è attingibile - come si diceva in precedenza - solo a partire da uno sguardo sinottico, che valorizzi i tratti della personalità del maestro non necessariamente funzionali all'esposizione delle idee ora di Senofonte, ora di Platone, ora di altri allievi; ma anzi messi in ombra o svalutati. Per questi motivi, il nucleo concettualmente più importante dell'opera di Rossetti mi sembra quello in cui, da Platone, da Senofonte e dagli altri socratici, egli estrae, per così dire, i tratti tipici e riconoscibili di Socrate e del suo modo di comunicare.

Questa fisionomia comunicativa di Socrate è analizzata nella parte centrale di Le Dialogue socratique. Essa emerge da situazioni comunicative ben identificabili e tipizzate, oggetto di un certo numero di unità dialogiche scritte da due o più autori di dialoghi socratici, l'analisi delle quali costituisce l'oggetto di tre saggi del volume; due di questi saggi 
partono da unità dialogiche contenute nei Memorabili di Senofonte: L'Euthydème de Xénophon (Cap. 2) e Savoir imiter, c'est connaître... (Cap. 3); e uno invece tratta l'argomento più in generale, Le ridicule comme arme entre les mains de Socrate et de ses élèves (Cap. 5).

$\mathrm{Nel} \mathrm{saggio} \mathrm{sull'Eutidemo,} \mathrm{è} \mathrm{analizzata} \mathrm{una} \mathrm{sezione} \mathrm{dialogica} \mathrm{dei} \mathrm{Me}$ morabili, la IV.2, in cui Senofonte ha il merito di farci vedere in che modo Socrate riusciva a suscitare nei propri interlocutori una reazione di profondo turbamento e di grandissima eccitazione. Sappiamo che anche Alcibiade era stato vittima di un tale sconvolgimento, ma esso è raccontato post eventum nel Simposio di Platone e più specificamente nell'Alcibiade di Eschine di Sfetto, mentre nel nostro testo viene mostrato l'intero processo nella sua tensione drammatica. Di più, questo capitolo, che Rossetti chiama Eutidemo a somiglianza del dialogo omonimo di Platone, a differenza delle altre sezioni dei Memorabili in cui Eutidemo è l'interlocutore di Socrate, è interamente incentrato sulla creazione di una situazione spiazzante per il giovane.

Socrate sceglie un approccio al giovane alquanto indiretto, in modo da non dichiarare apertamente le proprie aspirazioni a diventarne il maestro, per non compromettere la possibilità di conseguire l'obiettivo. Eutidemo, dapprima riottoso agli insegnamenti di Socrate, è presto coinvolto in un serratissimo dialogo col maestro e ridotto all'aporia con inusuale durezza. Egli, per tutta la durata della 'lezione' non viene mai rassicurato, per esempio su ciò che egli potrebbe non sapere di sapere, né gli viene mostrato l'obiettivo finale dell'agitazione creata. La tensione e l'insicurezza di Eutidemo giungono ad un punto di non ritorno, senza che si intraveda, nel corso dello scambio dialogico, un suo possibile riscatto. Nessun insegnamento positivo è fornito all'allievo, nessuna lezione nel senso tradizionale del termine, quanto piuttosto - argomenta Rossettiuna riflessione 'metacognitiva'. A Eutidemo è mostrato, in actu, cosa possa voler dire 'comprendere', cioè avere familiarità con «ensembles complexes de signifiés, d'usages linguistiques, de critères de jugement, de valeurs et de non-valeurs, de compatibilité et d'incompatibilité de positions déterminées, et d'autres choses encore». ${ }^{27}$

Una volta che l'allievo è diventato tale, che tipo di apprendimenti lo attende? Quali pratiche egli dovrà saper padroneggiare? superato il primo scoglio dell'impatto con Socrate, come si svolgeva poi la relazione?

${ }^{27}$ Rossetti 2011, p. 98. 
Su questo aspetto non ci sono molte testimonianza dirette, ecco allora la grande opportunità offerta dal capitolo III.8 dei Memorabili, da leggere insieme al passo dell'Apologia platonica che tratta lo stesso argomento: allievi che imitano Socrate e praticano l'elenchos: «une façon socratique de réfuter bien établie, connue comme telle et couramment qualifiée come elenchos». ${ }^{28}$ Il passo di Senofonte ci mostra qualcosa di più: infatti, si tratta di un allievo che vuole usare l'elenchos contro Socrate. La sfida diventa l'occasione per fornire una lezione davvero esemplare, sia all'ambizioso Aristippo sia agli altri astanti: il maestro si sottopone all'esame come rispondente e, avendo capito quale tipo di risposta l'allievo si aspetta, chiede spiegazioni supplementari per meglio comprendere, a suo dire, il senso della domanda; in realtà, per strappare all'allievo il ruolo di interrogante. Può così prendere forma l'impressione che «l'objectif poursuivi per Socrate est d'ordre relationnel, et non pas d'ordre cognitif ou ordonné à une recherche». ${ }^{29}$

Per fare ciò, tuttavia, Socrate è costretto a non seguire certe regole non scritte dell'elenchos, come Aristippo prontamente gli rimprovera nel suo secondo tentativo di esaminare il maestro. Tutto ciò presuppone una rete concettuale, un repertorio ben conosciuto, un tipizzazione di pratiche discorsive, di misure e contromisure e perfino una quantità di esempi padroneggiati e conosciuti dagli allievi; presuppone cioè che la pratica di imitare Socrate fosse in qualche misura diffusa e ben conoscibile.

Infine, veniamo ad un aspetto tipico del dialogo socratico: la ridicolizzazione dell'avversario. Essa consiste nel mostrare un tale contrasto - in un qualche tratto visibile dell'interlocutore - fra le apparenze e la realtà, la superficie e la sostanza, da scatenare una reazione incontrollabile, imprevedibile e irreparabile. Socrate (ma anche Platone) è maestro in materia di ridicolo: il dossier di testimonianze raccolte e commentate da Rossetti mostra come esso nasca dal procedimento tipicamente socratico di infondere nell'interlocutore la sicurezza, dapprima, e la speranza sempre più flebile, in seguito, di riuscire a sormontare la difficoltà creata da Socrate col suo elenchos; difficoltà che finiscono per sembrare all'interlocutore più grandi, non solo di quanto gli siano sembrate all'inizio della conversazione, quando gli si era fatto credere di essere un esperto in materia, ma anche di quanto in effetti non siano, in molti casi. Senofonte,

\footnotetext{
${ }^{28}$ Rossetti 2011, p. 107.

${ }^{29}$ Rossetti 2011, p. 112.
} 
nota Rossetti, rende a meraviglia l'atmosfera di asfissia intellettuale che Socrate riesce, non senza evidenti forzature, a instaurare.

Ma qual è la posta in gioco del mostrare l'inferiorità intellettuale degli interlocutori e i limiti della loro paideia, ed esporli talora crudelmente al ridicolo, minandone l'immagine di sé? La strategia - dice Rossettiserve a condurre l'interlocutore alla metanoia. Il grado di umiliazione inflitto all'interlocutore è funzionale a scatenare una crisi dalla quale egli possa uscire consapevole di un bisogno nuovo, di un ripensamento radicale, di un desiderio di cambiare a fondo la propria vita. Un simile dramma interiore può prodursi soltanto a seguito di una vera e propria scossa elettrica, che colpisce - è bene sottolinearlo- un interlocutore minimamente preparato a subirla.

A conclusione di questa sezione, il capitolo intitolato La rhétorique de Socrate (Cap. 6) fa il punto su una questione davvero spinosa: se esiste ed è riconoscibile un 'modo socratico' di organizzare l'interazione comunicativa, un modo estremamente raffinato e in grado di vincere vigorose resistenze e insinuarsi nelle convinzioni più profonde dell'interlocutore, scardinandole; esso è ascrivibile al dominio della 'retorica'? I commentatori moderni sono restii a usare questo termine, legato a simboli e valori lontani dalla ricerca della verità'. ${ }^{31}$ La retorica tuttavia non è soltanto un insieme di regole atte a garantire il successo di una strategia comunicativa. Molta parte di questo successo è infatti dovuta alla capacità del locutore di adattare continuamente i mezzi che egli intende dispiegare per raggiungere il suo fine, a ciò che di imprevedibile e mutevole si costruisce durante la comunicazione, che è sempre evento, cioè accade in un tempo, in una durata. Ciò spiega come una gestione sapiente del «flux communicationnel» abbia più peso, per il raggiungimento dell'obiettivo, di qualsiasi regola o codificazione preesistente. In questa gestione sapiente, questa l'idea di Rossetti, gioca un ruolo notevole la «retorica dell'anti-retorica», cioè l'esibizione di una rinuncia all'ornamento retorico, all'abilità oratoria (micro-retorica), a copertura della non rinuncia alla capacità di dibattere una questione, di ritorcere un argomento, smuovere un'emozione (macro-retorica). La sottovalutazione di sé, la deferenza nei confronti di alcuni interlocutori, una certa

${ }^{30} \mathrm{Vd}$. anche, sulle relazioni fra dialogo socratico e retorica, Candiotto 2012, pp. 65-80.

${ }^{31}$ Rossetti 2011, p. 219. 
artefatta ingenuità ed altre forme di modestia studiata sono stratagemmi per distogliere l'attenzione del lettore dai procedimenti retorici effettivamente messi in atto per raggiungere certi scopi e far passare certi enunciati. In tal modo la retorica diventa una sorta di dispositivo trasparente e inavvertito che dispiega i suoi effetti senza che l'interlocutore e il lettore possano prendere - in un primo tempo - adeguate contromisure (poi, forse, è già troppo tardi ed essi si trovano inestricabilmente implicati: «nous sommes encore sous l'effet de la 'rhétorique de la anti-rhétorique' socratico-platonicienne» ${ }^{32}$ ).

Si giunge così a concludere che -insieme al politico e all'imprenditore, al venditore, al pubblicitario, al commediante, al sacerdote, al medico e all'agente di cambio - nell'ampia categoria di coloro che arrivano a imporre le proprie idee con la fascinazione o con l'imbroglio, occorre mettere anche il filosofo.

Posto comunque che esiste una strategia macroretorica trasparente e inavvertita, Rossetti passa a analizzarla nel dettaglio identificandone, come tratti fondamentali, lo sforzo di influenzare gli interlocutori esercitando un controllo molto stretto sul proprio «univers intérieur» che non viene quasi mai manifestato; l'osservazione minuziosa dell'interlocutore e la valutazione accurata della sua recettività e sensibilità ad un certo coinvolgimento emotivo; la non minore attenzione agli interlocutori secondari e alla loro predisposizione ad identificarsi con l'interlocutore principale o con lo stesso Socrate; il controllo dell'interlocutore dall'interno, attraverso la messa in discussione dei suoi criteri, della visione di sé etc.; l'opera di prevenzione di reazioni di rifiuto o ostilità; l'inibizione della capacità e della facoltà dell'interlocutore di dirigere il corso del dialogo; la cura nel neutralizzare le difese e produrre una impasse.

In questa prima fase ha molta importanza quella che Rossetti definisce la 'formattazione' dell'interlocutore: cioè la preparazione, la creazione di una predisposizione, di un binario su cui far correre la comunicazione. Sapientemente 'formattato', l'interlocutore può essere 'saturato', fatto oggetto di una crescente sovra-stimolazione che non gli lasci il tempo di reagire. In seguito, dopo aver portato all'estremo la pars destruens, anche forzando impercettibilmente la mano e fornendo rappresentazioni distorte della realtà o vere e proprie bugie, la strate-

\footnotetext{
${ }^{32}$ Rossetti 2011, p. 224.
} 
gia socratica prevede che si lasci intravedere una possibile meta, una prospettiva nuova, un nuovo bisogno cui occorre dare soddisfazione. Ciò implica che, giunto a questo punto, l'interlocutore scopra anche la propria libertà e responsabilità nel riorganizzare le proprie idee e la propria condotta.

Un aspetto molto importante di questa strategia concerne l' «histoire personnelle» di Socrate: essa, sostiene Rossetti, è messa inizialmente a disposizione dell'interlocutore per indurlo a fidarsi del maestro Socrate; ma alla fine del percorso risulta chiaro che nulla di ciò che è stato detto o fatto nel corso del dialogo ha realmente importanza agli occhi del filosofo. Anzi, la qualità dell'argomentazione stessa è meno importante del clima generale, di fiducia e di riconoscenza, che è stato prodotto (ma anche modulato: perché c'è anche un ritmo della comunicazione da padroneggiare, alternando momenti di relax a momenti di pressione). Di più, Socrate non sembra mai soffrire personalmente quello choc emotivo che impone agli altri, non si lascia mai 'formattare' né 'saturare'.

Se la strategia macro-retorica è trasparente per l'interlocutore è invece visibile - per così dire - il ritaglio in negativo dei procedimenti micro retorici: nel senso che viene esibita la parola scarna e disadorna, il lessico ripetitivo e quotidiano, la banalizzazione, tutte scelte che dovevano esercitare un impatto fortissimo in una temperie culturale dominata dal professionismo dei sofisti.

La conclusione è sorprendente: la retorica di Socrate è ciò che conosciamo meglio del Socrate, come si diceva un tempo, "storico". La chiave d'accesso più sicura all'animus del filosofo.

Questa ci sembra anche la prospettiva più interessante del lavoro di Rossetti che, da gran conoscitore di strategie comunicazionali, la fa soltanto intravedere al lettore, fornendogli un solo indizio: il Fedro, il dialogo in cui Socrate apre realmente uno spiraglio sul proprio «univers interieur», sulle passioni che lo animano e dalle quali egli corre il rischio di essere dominato.

\section{Qualche questione}

F. Roustang, nell'Avant-propos ha ragione anche nel vedere uno dei punti di forza del volume nell'impertinenza con cui il discorso platonico, e non solo, viene interrogato; e nel precisare che a farlo non è solo lo specialista, con i suoi 'ferri del mestiere', ma anche il «lecteur impitoyable» 
(che qualche strumento ce l'ha anche lui). ${ }^{33}$ Questo mi conforta nella possibilità di porre qualche questione.

'Distillazione' e 'sindrome di Aristippo'. Nel dialogo platonico, il lettore è portato ad adottare un'attitudine di superiorità nei confronti dell'interlocutore - i vari Eutifrone, Ippia, Ione - e di complicità pur se riverente nei confronti di Socrate. Tale che anche il commentatore ha difficoltà ad accorgersi quando Socrate (anche lui!) ha torto. Bisogna fare, dice Rossetti, un lavoro di decondizionamento prima e di ripulitura dal filtro specifico del genere dialogico e della scrittura platonica, poi. Tuttavia: come essere certi che facendo questo lavoro di ripulitura il commentatore non sia ancora sotto l'effetto del dispositivo socraticoplatonico?

Alla fine del primo libro della Repubblica, dopo il violento scontro fra Socrate e Trasimaco, il lettore si è persuaso senza troppa fatica che Socrate - come di consueto- ha vinto sull'interlocutore, il quale è peraltro già condannato dalla sua antipatia e dai suoi modi violenti. ${ }^{34}$ Ma Platone ha bisogno che il lettore trovi da qualche parte lo stimolo ad andare avanti, molto avanti, nella ricerca e che lo segua per molti altri libri. Così fa dire a Socrate di essere insoddisfatto della risposta data a Trasimaco. ${ }^{35}$ Quella battuta, posta proprio in conclusione, ci fa immediatamente riguardare il dialogo con occhi nuovi, contro Socrate stavolta e ci richiama alla memoria tutte le volte in cui, in altri dialoghi Socrate, si è comportato nel modo che Trasimaco gli rimprovera. ${ }^{36} \mathrm{Nei}$ libri successivi, l'adesione a Socrate e alle idee che egli espone non è disgiunta dalla nuova attitudine critica verso le precedenti idee e modalità di dialogo!

${ }^{33}$ Roustang in Rossetti 2011, p. 17.

${ }^{34} \mathrm{Pl}$. Rep. $350 \mathrm{c}$.

35 Già a partire da Rep. 350d Platone aveva cominciato a seminare dei dubbi, che culminano nella battuta finale di Socrate: «Di sicuro non ho fatto un grande festino, ma per colpa mia, non tua», con cui, servendosi della metafora del banchetto e del ghiottone, Socrate fa autocritica e apre la porta alla grande costruzione/visione della nascita dello stato che occuperà i libri successivi.

${ }^{36}$ Questo scarto è stato notato da moltissimi studiosi ed utilizzato da Vlastos, in modo molto convincente, per argomentare sulla presenza di due Socrate - earlier e middle - nella Repubblica: Socrate, che nei ragionamenti contro Trasimaco ha ancora fiducia nella possibilità di scoprire la verità morale con la procedura investigativa elenctica, nelle righe conclusive del I libro è mostrato dubbioso e, a partire dal II libro abbandona il metodo. Vd. Vlastos 1998, pp. 59-105 e 331-334. 
Perché non pensare che, impercettibilmente, Platone lasci affiorare anche in altri dialoghi qualche elemento utile a fare nascere nel lettore il dubbio che altre e più idonee soluzioni possano essere date dei problemi che Socrate espone? In altre parole: e se fosse quella strategia macroretorica dotata di un dispositivo che mentre appaga il lettore non filosofo, introduce - spezzettando l'argomentazione, facilitando troppo certe vittorie, richiamando altre possibili risposte - 'semi' di insoddisfazione nel lettore filosofo?

Gli indici analizzati, questa la mia impressione, possono funzionare come voces mediae, di cui il lettore-lettore dà un'interpretazione 'convergente' e il lettore-filosofo una 'divergente'. Per esempio, quando un interlocutore risponde: «così sembra», la locuzione è in grado di funzionare da dispositivo che generi l'impressione di variatio rispetto al semplice «si» nel lettore già poco propenso ad esercitare un controllo rigoroso sull'argomentazione; e di dubbio nel lettore che invece legga il dialogo da filosofo, il quale si chiede: «così sembra?». Platone si comporterebbe come chi usasse scientemente una vox media lasciando che altri riempia in un modo o in altro la sua affermazione. ${ }^{37}$

Questo lettore-filosofo non farebbe altro che quello che già gli allievi di Socrate facevano: «mi imitano e perciò interrogano». La lunga consuetudine con Socrate porterebbe come frutto ultimo l'attitudine critica

${ }^{37}$ Per esempio, chi, davanti ad un regalo non gradito dicesse all'altro: «non dovevi assolutamente farlo!» potrebbe essere inteso in due modi differenti. Egli potrebbe aver usato una formula di cortesia, e allora il senso sarebbe: «non dovevi disturbarti», oppure potrebbe aver inteso dire effettivamente: «sarebbe stato meglio se non lo avessi fatto!» e in quel caso si dovrebbe interpretare la sua come un'amara constatazione. In una situazione comunicativa siffatta, il parlante intende offrire non tanto significati diversi di uno stesso enunciato, quanto differenti modi di concepire l'enunciazione: usando una formula di cortesia o una formula di assenso, il parlante rinuncia a compiere un atto di enunciazione proprio, perché ripete una 'formula' che in quanto tale ha un autore collettivo e anonimo e vale non (più) per il suo significato ma perché inserita in un'insieme di consuetudini linguistiche e di automatismi; ma il parlante può anche pronunciare o intendere quella formula, animandola, facendosene autore, mostrando di usarla per il suo significato in quella determinata situazione comunicativa: è il caso del lettore-filosofo, che (mentalmente) asserisce «così sembra» soltanto se ha effettivamente sottoposto il contenuto della proposizione che ha appena ascoltato/letto ad un esame di verosimiglianza; o del ricevente di un dono sgradito che emette un giudizio sull'opportunità dell'azione di cui è destinatario. Alcuni enunciati possono essere prodotti o recepiti sia come enunciati citazionali che non, ed ecco la possibilità di istituire due livelli diversi di lettura. Si tratta di casi di citazione, vd. su ciò, La Matina 2001. 
contro lo stesso Socrate: una sorta di 'sindrome di Aristippo' inoculata dal maestro per via di mimesi indotta, col suo ripetere instancabilmente il suo elenchos. Il commentatore che abbia dimestichezza col dialogo socratico è indotto a rivolgere l'arma dell'interrogazione socratica contro di esso, come l'Aristippo senofonteo nei confronti del maestro. Mi chiedo perciò se il compito della 'distillazione' non sia, in definitiva, un tipo molto raffinato di elenchos che il commentatore, conoscendo Socrate, sa riprodurre.

\section{La retorica}

La questione terminologica. Quando, nel trattare della retorica socratica, Rossetti fa luce sul fatto che nessuna comunicazione può essere interamente spiegata da regole date ante, illumina un aspetto essenziale della comunicazione stessa che ha che fare con la natura ambigua e circolare della regolarità. Quando afferma che, nell'esibire la sua estraneità ai procedimenti micro-retorici, Socrate di fatto "sta seguendo una regola" (una sorta di regola non scritta della retorica), Rossetti parla di regolarità come di qualcosa che si applica al comportamento altrui dall'esterno, sulla base di certi segni, linguistici e non, esibiti in modo più o meno enfatico. Questa dichiarazione di estraneità alla retorica era funzionale ad attirare l'attenzione dell'interlocutore sul comportamento linguistico dei retori e dei sofisti. In che cosa consiste quel comportamento? In comportamenti micro- e macro-retorici. In primo luogo nella loro pretesa di insegnare. Ma, obietta Socrate in vari luoghi, ciò che i sofisti fanno è soltanto incantare l'uditorio con parole fiorite, sollecitando una risposta empatica immediata ma superficiale, cosa che è sottolineata molto bene nel libro riguardo a Gorgia.

Sofisti e retori appaiono autorevoli, ma non lo sono. Le parole che ostentano, questo il punto a mio avviso, non hanno alcun corrispettivo in alcuna forma di vita, né propria né altrui. ${ }^{38}$ La questione che Socrate pone, rifiutando le procedure retoriche, potrebbe essere allora se la lingua sia un gioco colto o una forma di vita, se l'intentio comunicativa debba o no essere radicata in forme di vita piuttosto che in asserzioni.

\footnotetext{
${ }^{38}$ È anche possibile chiedersi in quale tipo di comunità si inserisca la comunicazione sofistica e quale essa a sua volta crei, con le aspettative che genera e le idee che veicola. Mi permetto di rimandare, su questi aspetti, a Caserta 2005.
} 
A dirlo bene è Lachete a conclusione di un passo giustamente famoso: «Quanto a Socrate, dei suoi discorsi non ho esperienza, ma, a quanto pare, ho prima sperimentato le sue azioni, e in esse l'ho trovato degno dei bei discorsi e di ogni franchezza». ${ }^{39}$ Allora mi sembra che il discrimine sia questo: finché si dice che l'importante non è parlare ma agire, si è nell'ambito della retorica (un dire rimpiazza l'altro); se oltre a dirlo o invece che dirlo si agisce effettivamente, si è fuori dalla retorica (un fare invera il dire), non si segue alcuna regola ma semmai la si incarna (o addirittura la si inventa se quell'azione non era mai stata censita prima come utile a conseguire il successo in ordine a un certo fine). Il discrimine non riguarda, credo, il fine della comunicazione governata dai procedimenti retorici, quanto il rapporto che lega il parlante alla sua enunciazione, la responsabilità che si assume riguardo ad essa: è una tecnica - quella di Socrate - ma anche di più, un modo di vivere. Se Socrate dice di volersi dedicare all'educazione dei giovani attraverso la synousia e la cura, è possibile che sia (macro o micro) retorica; ma quando veglia col giovane e ansioso Ippocrate in attesa che giunga il giorno, e in qualche modo ci è mostrato che lo ha fatto effettivamente, non è più solo un gioco linguistico, più o meno serio. A meno di non voler definire 'retorico' tutto il fare comunicativo orientato ad uno scopo: osservare, recarsi in un certo luogo, sedersi o alzarsi, camminare, stare fermo eccetera.

In effetti, Rossetti si pronuncia in favore di un ampliamento del campo di applicazione della retorica: «À moins de penser que la rhétorique ne s'occupe pas de ce qui est décisif pour le succès de la comunication, il est donc impératif d'élargir son champ d'observation à tout procédé visant à assurer ce succès et apte à le faire, fût-ce aux dépens de la distance critique des destinataires»..$^{40} \mathrm{Ma}$, io ritengo, porre la questione in questi termini significa dire che finché non c'è il successo/insuccesso non si può dire che una certa azione sia finalizzata o meno ad ottenerlo. È il successo comunicativo a dirci se Socrate, agendo in un certo modo, sta o non sta seguendo una regola o un insieme di regole? Oppure, quelle azioni scaturiscono dalla reazione all'interlocutore di un locutore, quale Socrate, che ci si presenta come qualcuno che ha trasferito nella sua persona, cioè nelle sue azioni, un progetto comunicazionale?

\footnotetext{
${ }^{39}$ Plat. Lach. 188c-d.

${ }^{40}$ Rossetti 2011, p. 221.
} 
Se dunque la rinuncia all'ornamento retorico corrisponde ad una analoga rinuncia all'azione detta in favore dell'azione effettivamente compiuta, e se questa azione fatta eccede il solo raggiungimento del successo comunicazionale per configurarsi come profonda disposizione personale, credo che 'retorica' sia troppo poco, per quel suo etimo che, rimandando comunque a un dire, non descrive che una parte di ciò che avviene nel dialogo.

Il coinvolgimento di Socrate. Un aspetto molto importante della strategia socratica concerne, sostiene Rossetti, il fatto che nulla di ciò che è stato detto o fatto nel corso del dialogo ha realmente importanza agli occhi del filosofo: Socrate non sembra mai soffrire personalmente quello choc emotivo che impone agli altri, non si lascia mai 'formattare' né 'saturare'. Questo è indubbiamente vero nella maggior parte dei casi, ma non in tutti. Non mi riferisco al Fedro, che lo stesso Rossetti richiama come eccezione, ma al fatto generale che, se il timone dell'evento comunicazionale è saldamente nelle mani del filosofo, i venti e le correnti che egli cerca di governare provengono dall'interlocutore. Questi venti e queste correnti o, fuor di metafora, ciò che costituisce la peculiarità dell'interlocutore, determina il modo in cui Socrate si comporta (e talora il suo stesso decidere di agire), che è dunque reazione emotiva ad una novità personale e individuale non meno che adattamento ad una situazione.

Quando Senofonte ci racconta l'approccio di Socrate ad Eutidemo, non ci presenta la decisione di Socrate di avvicinarlo come una reazione in termini di identificazione empatica con l'impasse del giovane? Un'adesione emotiva al suo conflitto interiore fra estraneità culturale ai coetanei e sostanziale condivisione con essi di una medesima effettiva condizione. Naturalmente, possiamo anche leggere la scena solo come una sorta di scena di caccia, in cui un cacciatore scorge una preda afflitta da una debolezza e sceglie di attaccarla, valutando le possibilità alte di successo, ma così facendo riduciamo ogni relazione umana a relazione di potere, facendo di Socrate una sorta di prototipo di homo strategicus hobbesiano.

Lo stesso non accade con Aristippo? Che significa che nei confronti dell'impertinente allievo Socrate decide di «fare ciò che deve»? Se non che su un astratto desiderio di avere la meglio nel ragionamento, egli fa valere la sua immedesimazione nel tormento del giovane, che desidera alleviare? Che altro senso può avere precisare che Socrate agisce in quel 
modo perché «sa che quando qualcosa ci tormenta abbiamo bisogno di qualcosa che lo faccia smettere»? E chi è quel «noi», se non noi tutti, incluso Socrate che infatti «sa» cosa agita il giovane allievo?

Seppure non si lascia scioccare, non si può escludere - stando alla rappresentazione che ne viene data - che certe situazioni particolari di sofferenza personale e di impasse non tocchino certe corde emotive di Socrate o forse non risveglino antichi analoghi tormenti. Ł̀ anzi possibile che le speranze e i libri di Eutidemo smuovano la memoria dell'esperienza analoga raccontata nel Fedone: di speranza destata nel giovane Socrate dai libri di Anassagora, di desiderio disperato di «farsi discepolo di chiunque» gli avesse insegnato quello che cercava e di persuasione, poi, di dover impastare tutto un altro metodo, volgendosi ai discorsi non meno che alla realtà. ${ }^{41} \mathrm{E}$ che dunque la decisione del maestro di farsi avanti con Eutidemo e di procedere in un certo senso (di metodo, appunto, e non di spiegazione) sia frutto di questa risonanza emotiva, non meno che elemento di una strategia.

Almeno in questo caso, la formattazione dell'interlocutore a recepire il messaggio di Socrate riposa su una precedente formattazione di Socrate a riconoscere esattamente la condizione di colui in cui si imbatte.

Durante simili conversazioni, però, Socrate non subisce saturazione, non è mai preda di tensione insostenibile. Nel Protagora, c'è una sezione di elenchos molto simile a quella condotta da Aristippo contro Socrate: Socrate chiede se Protagora affermi l'esistenza di «cose buone»; il sofista, irritato e sulla difensiva, fornisce una risposta lunga che blocca l'elenchos di Socrate e che dimostra l'esistenza di cose buone che talvolta sono dannose, proprio come intendeva fare Aristippo per avere la meglio su Socrate; e proprio come fa Socrate quando prende il controllo del dialogo con il suo allievo nel passo senofonteo. A questo punto, nel Protagora, Socrate fa come Aristippo! Cioè richiama l'altro all'osservanza delle regole e, quando questi non vuole farlo, si alza per andar via (come Eutifrone!), con la scusa di un impegno. ${ }^{42}$

Se lo sbocco della situazione è diverso (Socrate non si converte ad alcunché), la saturazione è la stessa che di norma il filosofo impone ai suoi interlocutori: Socrate ha la sensazione di non poter stare dietro

${ }^{41}$ Plat. Phaedo 96a-100a. Su questo 'intermezzo autobiografico', vd. Cerri 2012, pp. 151-194.

42 Plat. Prot. 334a-335d. 
a Protagora e che continuare così, disperdendo energie in uno scontro frontale (un «agone di discorsi», come piace al sofista), non sarebbe nel suo interesse né nel suo stile. A Callia, che vuole trattenerlo, dice: «è come se tu mi chiedessi di star dietro al corridore Crisone di Imera nel pieno della sua forma, o di gareggiare e tener dietro a uno di quegli atleti che corrono per lunghe distanze o per un'intera giornata, ma io (...) non posso correre velocemente». ${ }^{43} \mathrm{La}$ similitudine con la corsa rende bene l'idea del debito d'ossigeno, della sovra-stimolazione, dell'asfissia mentale che il comunicare di Protagora gli provoca.

Infine, il fine. Al di là del gioco di parole: il fine di tutta una serie di manovre messe in atto da Socrate è la conversione dell'interlocutore, la scossa elettrica che aspira a fargli sentire il bisogno di cambiare vita, di riorganizzare le proprie idee. Ma: non c'è alcun fine che riguardi Socrate? La sua, di conversione, è compiuta una volta e per tutte? Nel Fedro, di cui nel libro è sottolineata l'eccentricità, Socrate dice di sé:

non sono ancora in grado di conoscere me stesso come prescrive l'iscrizione delfica. Mi sembra perciò ridicolo, finché ignoro ancora ciò, prendere in considerazione problemi che mi sono estranei (...) esamino non queste, ma me stesso, per scoprire se per caso io sia una fiera più complicata e più fumosa di Tifone, o se io sia un animale più mansueto e più semplice, partecipe per natura di una sorte divina e senza fumo. ${ }^{44}$

L'immagine di Tifone, che sembra ripresa da un brano di Pindaro sull'Etna, rimanda ad una problematica che non è solo del Fedro, ma che si trova per esempio in un frammento di Diogene Laerzio sull'incontro fra Socrate e Zopiro, e chiama in causa, con la fisiognomica, il rapporto fra bruttezza/mostruosità e conoscenza di sé (ed eventualmente, educazione di sé). ${ }^{45} \mathrm{Il}$ tema dell'incontro con Zopiro testimonia anche come il

${ }^{43}$ Plat. Prot. 335e- 336a.

44 Plat. Phaedrus 229e-239a. Il mito di Tifone era narrato da numerose fonti arcaiche che collocano la Tifonomachia come ultimo episodio della lotta fra Zeus e le forze che minacciano l'ordine olimpio; anche l'arte figurata documenta la lotta mostrando Tifone con due teste e due o più code di serpente in luogo delle gambe: il cenno di Socrate alla natura "complicata" ( $\left.\pi \circ \lambda \hat{v} \pi \lambda \circ \varkappa_{0 \varsigma}\right)$ di Tifone potrebbe alludere alla natura composita del suo aspetto e all'aspetto "rugoso" del suolo vulcanico. Mi permetto di rimandare, per un'analisi di questi temi, a Caserta 2012, pp. 167-189.

${ }^{45}$ In un noto aneddoto riportato da Diogene Laerzio II.105, Socrate si imbatte in un tale Zopiro, un mago giunto dall'oriente che si dichiara esperto di fisiognomica. Sen- 
problema della conoscenza di sé fosse un problema che Socrate poneva a se stesso e non solo ai suoi interlocutori. Allora occorre chiedersi: in che modo l'altrui scossa elettrica, l'altrui conversione si articola rispetto alla conoscenza di sé, per lo stesso Socrate? Che cosa dice o non dice rispetto a questo, rispetto all'essere egli Tifone o una semplice creatura partecipe di sorte divina, il fatto di portare altri alla conversione? Cioè, ancora: la relazione dialogica, con quello che essa significa in termini di contesto etc., quale aspetto dell'identità personale di Socrate consente allo stesso Socrate di conoscere, sottoporre a verifica e governare mentre dialoga?

Oscurità di Platone, oscurità di Senofonte. La retorica di Socrate è documentata anche dai socratica senofontei, sia al livello di macroche di (assenza di) micro-retorica. Per di più, reticenze, discontinuità dell'argomentazione, obliquità di certi enunciati, forzature, 'zone oscure' esistono, oltre che in Platone, anche in Senofonte. Messaggi insondabili e (finora) inspiegabili. Non si può certo dire, però, per Senofonte che l'obliquità o anche il 'melange' di contesto e punti di dottrina abbia uno scopo protettivo. Si ha invece buon gioco, come commentatori, ad addebitare all'imperizia di Senofonte tutto ciò che non quadra e non fila! Motivo per il quale raramente ci si preoccupa di un possibile «côté paraénonciatif» del dialogo di Senofonte. ${ }^{46}$

A meno che non si voglia addebitare in parte a Socrate la costruzione di questo filtro. Allora si dovrebbe prendere sul serio anche ciò che di solito si liquida come 'farina del suo (di Senofonte) sacco'. In Mem. III 8, per esempio, si può osservare una formulazione generale, piatta e banale che in genere viene attribuita al solo Senofonte. Essa si trova a corollario dell'elenchos rivolto al maestro dall'allievo impertinente, e

za aver mai avuto a che fare con il filosofo, basandosi soltanto sul suo aspetto físico, Zopiro gli diagnostica una grande quantità di vizi e difetti. L'aneddoto si trova pure in Aulo Gellio II.18; Cic. De fato 5.10 e Tusculanae IV.80. Un dialogo intitolato Zopiro è collegato nella tradizione al nome di Fedone di Elide, reso famoso da Platone come narratore del dialogo che contiene la scena della morte di Socrate. Sulla scena dell'incontro tra Zopiro e Socrate, vd. Kahn 2008, p. 19; Rossetti 1980; Giannantoni 1990 (SSR IV), pp. 115-119.

${ }^{46}$ Una tale direttrice di ricerca mi sembra assente dalle tendenze documentate negli ultimi decenni di studi senofontei, per un profilo dei quali, vd. Stavru-Rossetti 2010, pp. 25-35. Anche gli studi di impostazione più tradizionale sullo stile senofonteo si limitano spesso a lamentare una sostanziale superficialità di pensiero e monotonia di stile, a partire dall'ormai classico Gautier 1911. 
costituisce una 'lezioncina' di Socrate su come sia meglio edificare le case. Rossetti nota giustamente che essa lascia perplessi e aggiunge che in questo modo Socrate perde il tratto identificante che aveva avuto nella sezione immediatamente precedente. Nella quale, si è detto, Socrate aveva mostrato all'allievo Aristippo, come cavarsi dall'impiccio di un elenchos molesto, ora ribaltando il ruolo da rispondente a interrogante, ora rispondendo in modo tale da bloccare l'elenchos stesso. Con la lezioncina di urbanistica, nota Rossetti, si dissolve fino a scomparire la relazione con l'interlocutore e infine sparisce l'interlocutore stesso.

E se invece si trattasse di una zona 'oscura', nel senso di reticente e allusiva? L'immagine della casa che Socrate ritiene più gradevole da abitare e più pratica in ogni stagione è una casa più alta nella parte esposta a mezzogiorno, in modo da poter godere maggiormente del sole invernale, e più bassa nella parte esposta a nord, in modo da essere meno battuta dai venti freddi. Ma questa casa, che espone una piccola superficie all'ostilità (climatica) e una maggiore ai vantaggi (sempre climatici) non è forse come il filosofo quando, come ha ora fatto Socrate, non offre il fianco all'elenchos altrui e invece approfitta del vantaggio quando questo si presenta? Si riconoscerà che è una casa altamente strategica $\mathrm{e}$ intelligente.

Se si riconosce un valore allusivo al piccolo insegnamento urbanistico, nulla ci vieta di cercare analoghe allusioni anche da altre parti, per esempio nel contenuto dei contro esempi, a partire da quello con cui Socrate blocca il secondo tentativo di elenchos di Aristippo. Egli aveva chiesto a Socrate se conoscesse qualche cosa bella, Socrate risponde di conoscerne molte fornendo come esempio la 'diversità' fra un uomo bello nella corsa e uno nel pugilato, e fra uno scudo da difesa e una freccia adatta ad essere scagliata da lontano. ${ }^{47} \mathrm{Si}$ può pensare, io ritengo, che la menzione del pugilato e del dardo veloce contengano un riferimento allusivo al polýtropos Odisseo, pugile e tiratore con l'arco, ed alla sua metis; cioè in definitiva ad una capacità strategica di farsi cavo e pieno, basso e alto, vicino e lontano in relazione all'avversario del momento, proprio come la casa ideale! (Non sorprenderà trovare che, quasi sempre, la menzione della corsa e del pugilato, come discipline antitetiche, richiama modelli di comunicazione e di dialogo alternativi: frontale e con 'discorsi lunghi' la prima; obliqua e con scambio rapido di domanda

${ }^{47}$ Xen. Mem. III.8.4. 
e risposta, la seconda; lo stesso vale per la contrapposizione scudo/frecce, quasi sempre in contesti 'odissiaci'). ${ }^{48}$

Se così fosse, si dovrebbe riflettere sull'allusione e la reticenza in Senofonte, recuperando all'analisi una serie di passi finora (troppo?) semplicemente rubricati come espressione dell'insipienza del loro autore. ${ }^{49}$ C'è di più, perché questi corollari 'oscuri', a differenza di passi altrettanto mal interpretabili ma inseriti in un contesto macroretorico più saldo, si prestano più facilmente, qualora si perda cognizione dell'argomentazione più ampia in cui erano originariamente inseriti, ad essere estratti, antologizzati, ritagliati e fruiti in modo autonomo come kreiai, apoftegmi etc. L'allusività a livello di enunciato potrebbe allora contribuire a spiegare il diverso e sfavorevole destino di recit aneddotici -incastonati in recit altrui o persi - rispetto a unità dialogiche complesse, meglio conservate.

Da ultima, una suggestione. Il nucleo forte del 'Socrate in azione' è costituito da una serie di scene fortemente tipizzate. L'Eutidemo di Senofonte è per esempio un modello di "prima lezione", mentre l'elenchos rivolto da Aristippo a Socrate costituisce una scena che potremmo definire "l'allievo imita Socrate". La terza scena tipica che emerge dall'analisi di Rossetti potrebbe essere: "Socrate ridicolizza l'avversario". Valorizzando, come Rossetti ha fatto, ${ }^{50}$ alcune affermazioni di Alcibiade nel Simposio di Platone, si possono paragonare queste situazioni comunicative tipizzate a dei canovacci o piuttosto a degli spartiti musicali, di cui era possibile dare esecuzione - in tanti modi differenti, con più o meno successo, con un maggiore o minore grado di riconoscibilità- e di cui solo lo spettatore poteva riconoscere la fedeltà ad un originale. Naturalmente questo 'originale' non va inteso come evento specifico, storico, da replicare ma come insieme di tratti personali e singolari di Socrate, che l'esecuzione poteva più o meno intensamente evocare.

L'uso, sia da parte dei Socratici che da parte del nostro Autore, di alcuni termini — «scene», «formule», «esecuzione»- ci induce a chiederci se lo stesso Socrate non fosse consapevole di presentare il suo insegnamento in termini di performance aedica, e se gli studi socratici non possano

\footnotetext{
${ }^{48}$ Mi permetto di rimandare a Caserta 2013, in c. di s.

${ }^{49}$ Sulla pratica dell'allusività come strategia di senso, vd. Jullien 2011.

${ }^{50}$ Rossetti 1991, p. 24. Vd. anche www.rossettiweb.it/public/livio/doc/aspettidellaletteratura.pdf 102-107.
} 
allora trarre giovamento dalla filologia omerica e dai suoi metodi. Si leggano per esempio le parole con cui Jesper Svenbro descrive i risultati delle ricerche di Milman Parry, il quale: «mostrò come l'organizzazione formulare dei canti omerici fosse l'impronta irrefutabile di una lunga tradizione orale. I testi pervenutici, così letterari in apparenza, devono di fatto essere considerati come deviazione da una tale tradizione. Quel che noi oggi possediamo sono i testi di una Iliade e di una Odissea che, nella tradizione orale, erano canti multiformi». ${ }^{51}$ Esse potrebbero essere usate anche per descrivere le modalità di produzione e diffusione dei logoi sokratikoi! Che nel dialogo socratico una fase orale, non sappiamo quanto lunga, dovette precedere la fissazione per iscritto sembra confermato dalle analisi di Rossetti sulle testimonianze dei Socratici; ciò potrebbe essere anche desunto - secondo la suggestione che mi permetto di indicare - sia dall'organizzazione interna del testo, per la presenza in esso di scene tipiche, ripetizioni, esempi e contro esempi fissi se non formulari; sia dalle modalità di apprendimento da parte degli allievi che, come l'Iscomaco di cui ci parla Plutarco, sapeva proporre agli ascoltatori che lo chiedessero «un piccolo seme e saggio dei suoi <di Socrate> discorsi». ${ }^{52}$ Iscomaco si comportava dunque in modo forse analogo all'aedo omerico che, ci dicono i filologi e gli oralisti, sapeva combinare le sue formule tradizionali in presenza dell'uditorio. Egli doveva aver conseguito una preparazione specifica consistente in un lungo periodo di familiarizzazio$n e$, piuttosto che di insegnamento sistematico, che gli desse la padronanza delle formule (e dei segreti?) del dialogare socratico, in modo da saperle combinare, maneggiare, sostituire senza sforzo (la tipizzazione era infatti un modo per favorire e agevolare questa padronanza).

Niente di più che un'analogia, benché suggestiva, fra le modalità di produzione e diffusione dell'epos omerico e quanto, delle abitudini comunicative di Socrate con i suoi allievi, ci mostra l'interpretazione comunicazionale del logos sokratikos.

${ }^{51}$ Svenbro 1984, p. 31.

52 Pl. Mor. 516c. su cui vd. Rossetti 1991. L'analogia spiegherebbe anche il proliferare di varie unità dialogiche sul medesimo tema, poiché era tipica appunto dell'esecuzione aedica la possibilità di dare vita a versioni diverse di una stessa scena o canto, intendendo per identità una certa stabilità tematica. Accanto a questa modalità, quella che invece parte da un testo scritto potrebbe essere paragonata alla pratica rapsodica: Socratici che apprendono una versione di un testo, dipendono dalla parola scritta, dalla stabilità materiale del testo, come il rapsodo omerico. 
